\title{
Clinical Tick Borne Encephalitis in a roe deer (Capreolus capre- olus L.)
}

\author{
Graziana Da Rold ${ }^{1,2^{*}}$, Federica Obber ${ }^{1,2}$, Isabella Monne ${ }^{2,4}$, Adelaide Milanii ${ }^{2,4}$, Silvia Ravagnan ${ }^{2,5}$, Federica \\ Toniolo $^{2,5}$, Sofia Sgubin ${ }^{2,5}$, Gianpiero Zamperin ${ }^{2,4}$, Greta Foiani ${ }^{2,6}$, Marta Vascellari'2,6, Petra Drzewniokova ${ }^{2,3}$, \\ Martina Castellan ${ }^{2,3}$, Paola De Benedictis ${ }^{2,3}$ and Carlo Vittorio Citterio ${ }^{1,2}$
}

1 U.O. Ecopathology SCT2-Belluno, Istituto Zoprofilattico Sperimentale delle Venezie (IZSVe), Via Cappellari 44/A, 32100 Belluno,(BL, Italy);

2 OIE Collaborating Centre for Diseases at the Animal/Human Interface, Istituto Zooprofilattico Sperimentale delle Venezie (IZSVe), Viale dell'Università 10, 35020 Legnaro (PD, Italy);

3 Laboratory for Viral Emerging Zoonoses, Istituto Zooprofilattico Sperimentale delle Venezie, Viale dell'Università 10, 35020 Legnaro (PD, Italy)

4 Laboratory for Viral Genomics and trascriptomics, Istituto Zooprofilattico Sperimentale delle Venezie (IZSVe), 35020 Legnaro (PD, Italy)

5 Laboratory of Parasitology Micology and Sanitary Enthomology, Istituto Zooprofilattico Sperimentale delle Venezie, Viale dell'Università 10, 35020 Legnaro (PD, Italy)

6 Histopathology Laboratory, Istituto Zooprofilattico Sperimentale delle Venezie, Viale dell Universita 10, 35020 Legnaro( PD, Italy)

* Correspondence: gdarold@izsvenezie.it;

\begin{abstract}
Tick-borne encephalitis virus (TBEV) is the causative agent of Tick borne encephalitis, a severe zoonosis occurring in the Palearctic region mainly transmitted through Ixodes ticks. In Italy, TBEV is restricted to the north-eastern part of the country. This report describes for the first time a case of clinical TBE in a roe deer. The case occurred in the Belluno province, Veneto region, an area endemic for TBEV. The affected roe deer showed ataxia, staggering movements, muscle tremors, wide-base stance of the front limbs, repetitive movements of the head, persistent teeth grinding, hypersalivation and prolonged recumbency. An autopsy revealed no significant lesions to explain the neurological signs. TBEV RNA was detected in the brain by real-time RT-PCR, and the nearly complete viral genome (10,897 nucleotides) was sequenced. Phylogenetic analysis of the gene encoding the envelope protein revealed a close relationship to TBEV of the European subtype, and $100 \%$ similarity with a partial sequence (520 nucleotides) of a TBEV found in ticks in the bordering Trento Province. The histological examination of the midbrain revealed lymphohistiocytic encephalitis, satellitosis and microgliosis, consistent with a viral etiology. Other viral etiologies were ruled out by metagenomic analysis of the brain. This report underlines for the first time the occurrence of clinical encephalitic manifestations due to TBEV in a roe deer, suggesting that this pathogen should be included in the frame of differential diagnoses in roe deer with neurologic disease.
\end{abstract}

Keywords: roe deer, Tick-Borne Encephalitis, neurologic disease, pathology, genetic characterization

\section{Introduction}

Tick-borne encephalitis virus (TBEV), the causative agent of the severe and even lethal zoonosis Tick Borne Encephalitis (TBE), is a member of the genus Flavivirus, family Flaviviridae; the viral genome is a positive-sense, single-stranded RNA molecule of about $11 \mathrm{~Kb}$. Nowadays, five subtypes of TBEV are known, phylogenetically classified and characterized by different geographical distribution and severity of disease: European (TBEV-Eu), Siberian (TBEV-Sib), Far Eastern (TBEV-FE), Baikalian (TBEV-BKl) and 
Himalayan (TBEV-Him) [1-2]. The prevailing subtype in Western Europe is TBEV-Eu, the least virulent subtype with a case fatality rate of less than $2 \%$ compared to the TBEV-FE (20-60\%) and TBEV-Sib (7-8\%) [3-4].

TBEV is mainly transmitted through bites of infected ticks of the genus Ixodes to different mammals, including humans [5-6]. Occasionally, human infection can also occur via alimentary route in people consuming unpasteurized milk from infected dairy cattle or small ruminants [7]. Ixodes ricinus is the main vector of TBEV-Eu, while TBEV-Sib and TBEV-FE are mainly transmitted by Ixodes persulcatus. Ticks act as the vector and a reservoir of TBEV, which is transmitted from viraemic competent host animals to ticks [8] or by infected to uninfected ticks co-feeding on the same host [9-10-11]. Notably, infected ticks can transmit the virus to their offspring through transovarian transmission [12].

The ecology and epidemiology of TBEV depend on different interconnected factors such as climate, landscape and density of ticks and their hosts, including both species that are competent for TBEV transmission (as small rodents) and other species (e.g. wild ungulates) playing a key role in maintaining a consistent tick population, although not competent for viral transmission [13]. For this reason, natural foci of TBEV have a patchy distribution, ranging from few square metres to several square kilometres [14-15].

The actual impact of TBEV in mammals other than humans is poorly investigated. Indeed, few reports of clinical TBE in animals are available in the literature [16-24] and the infection is notably asymptomatic in ruminants, with few exceptions [25-28]. No clinical cases have been reported in roe deer so far, although this species is one of the most abundant cervids in Europe and acts as preferential host for I. ricinus [29-30]. In roe deer, viraemia is generally low and short, so that it is considered unable to infect ticks [31-36]. On the other hand, serological monitoring in this species has been used as a sentinel for TBE foci detection [36].

In the present paper, we describe clinical signs, pathological findings and the viral characterization in a case of TBE in a roe deer in Belluno province (Veneto region, North Eastern Italy), a longtime recognized endemic area accounting for about $40 \%$ of all the Italian cases of TBE in humans.

\section{Materials and Methods}

\subsection{Case history, clinical signs and autopsy findings}

On June $2^{\text {nd }} 2021$, the Belluno Provincial Police found a one-year-old female roe deer in the Belluno municipality, in a location named Modolo (4607'58.0"N; 12²15'11.3"E; 390 $\mathrm{m}$ a.s.1.). The animal was in poor general condition and showed various neurological signs including ataxia, staggering movements, muscle tremors, wide-base stance of the front limbs, repetitive movements of the head, persistent teeth grinding, hypersalivation and prolonged recumbency (videos are provided as supplementary material as VideoS1 and Video S2). Due to the severity of the clinical manifestation and the passive surveillance plans undertaken in the region to rule out infectious diseases of public health interest, the animal was therefore humanely culled and promptly submitted to the Istituto Zooprofilattico Sperimentale delle Venezie (IZSVe) for post-mortem examination and diagnosis.

An autopsy revealed no significant lesions to explain the neurological signs. Besides evident teeth wear presumably due to grinding, other findings were nonspecific. Concerning the brain, only mild vascular congestion was observed.

Seventeen engorged ticks, morphologically identified as adult Ixodes ricinus, were found in the skin and collected for molecular analyses to assess for the presence of common pathogens of public health interest. Roe deer's brain was collected for virological and histological investigations.

\subsection{Virologic and molecular testing}


First, rabies virus infection was ruled out through the direct fluorescent antibody (DFA) test coupled with real time PCR (RT-PCR) and virus isolation attempt [37-38]. A iQ Check Listeria monocytogenes II kit (Certificate number: BRD 07/10 - 04/05) was also used to detect DNA of L. monocytogenes. Based on negative results, the presence of a Flavivirus infection was therefore investigated through a specific RT-PCR targeting the 3' non coding region of the TBEV genome [39].

Nucleic acids were extracted from tick samples using the All Prep DNA/RNA mini Kit (Qiagen, Valencia, CA, USA), according to the manufacturer's instructions. DNA was amplified by SYBR Green real-time PCR (rPCR) assays for Borrelia burgdorferi (s.1.), Rickettsia spp., Babesia spp., and Anaplasma phagocytophilum; RNA was amplified by a specific real-time PCR (rRT-PCR) for TBEV [39-40].

\subsection{Illumina MiSeq sequencing, bioinformatics analysis and phylogeny}

Total RNA was extracted from brain tissue with the QIAamp Viral RNA mini kit (Qiagen) and then subject to double stranded cDNA synthesis using the Maxima H Minus Double-stranded cDNA synthesis kit \#K2561 (ThermoFisher), purified with magnetic beads (Agencourt AMPure XP, Beckman Coulter), and quantified with the Qubit dsDNA HS assay kit (ThermoFisher). Library preparation was performed using Nextera XT DNA sample preparation kit and processed on an Illumina MiSeq instrument with MiSeq reagent kit V3 (2x300 bp paired-end [PE] mode; Illumina, San Diego, CA, USA)

Illumina reads quality was assessed using FastQC v0.11.2 (https://www.bioinformatics.babraham.ac.uk/projects/fastqc) and consensus sequence of the whole genome obtained using the pipeline as detailed in Appendix A.

Whole genome consensus sequence and the nucleotide portion coding for the envelope protein (E) were compared with the most related sequences available in GenBank database. A multiple nucleotide sequences alignment for E containing the aforementioned sequences (21RS1767 and blast results) as well as representative ones for the five subtypes described so far TBEV-Eu, TBEV-Sib, TBEV-FE, TBEV-Bkl and TBEVHim was obtained using MAFFT v. 7 [2, 41-44]. A maximum likelihood phylogenetic tree was obtained using GTR+F+I+G4. To assess the robustness of individual nodes of the phylogeny, 1000 bootstrap replicates were performed. Phylogenetic tree was visualized using FigTree v1.4.3.

Envelope protein (E) and non structural protein 5 (NS5) amino acid sequences have been inspected to look for major viral determinants of virulence previously identified [45].

\subsection{Histological examination, immunohistochemistry and immunofluorescence}

A sample of midbrain collected at autopsy was fixed in $10 \%$ neutral buffered formalin, processed routinely, embedded in paraffin, microtome-sectioned, stained with hematoxylin and eosin (H\&E) and mounted on glass slides for histologic examination under optic microscope. Immunohistochemistry (IHC) for Listeria monocytogenes and Toxoplasma gondii was performed on further $4 \mu \mathrm{m}$ formalin-fixed paraffin-embedded sections in an automated immunostainer (Discovery Ultra; Roche, Ventana Medical Systems ${ }^{\mathrm{TM}}$ ). After dewaxing, sections were submitted to antigen retrieval with ULTRA Cell Conditioning Solution ( $\mathrm{pH}$ 6.0, Ventana) at $91^{\circ} \mathrm{C}$ (24-32 minutes). Sections were incubated with polyclonal rabbit antibodies, anti-Listeria $\mathrm{O}$ (1:500 diluted for 32 minutes, BD Difco ${ }^{\mathrm{TM}}$ ) and anti-Toxoplasma gondii inflammatory profilin (1:50 diluted for $40 \mathrm{~min}$, Clinisciences $^{\mathrm{TM}}$ ) at room temperature. The OmniMap anti-rabbit HRP (Ventana) was used as detection system. Sections of bovine and feline brain naturally infected by Listeria monocytogenes and Toxoplasma gondii, respectively, were used as positive controls. Replicate tissue sections, submitted to the same protocol without the primary antibody, were used as negative controls. 
For immunofluorescence analysis of the active microglia in the roe deer midbrain, 4 $\mu \mathrm{m}$-thick sections were re-hydrated and antigen retrieval was performed by incubation in citrate buffer $0.01 \mathrm{M} \mathrm{pH} 6$ at $95^{\circ} \mathrm{C}$ for 20 minutes. Slides were then permeabilized for 20 min at RT with PBS 1\% Triton X-100. Slides were saturated with Blocking Buffer (BSA 5\% in PBS $0.1 \%$ Triton [PBSt]) for 1 hour and incubated overnight at $4{ }^{\circ} \mathrm{C}$ with the mouse monoclonal anti-Iba1 (ionized-calcium binding protein 1) primary antibody (1:100 diluted, Abcam). The following day, sampled were incubated in the dark 2 hours with a secondary antibody (Goat anti-mouse Alexa Fluor 568, Thermo Fisher Scientific) conjugated with a fluorophore, previously diluted in 1\% BSA in PBSt. Slides were washed and mounted in with Fluoroshield Mounting Medium with DAPI (Sigma), lo label cell nuclei. Images were acquired with Leica TCS SP8 confocal microscope equipped with a CCD camera using LAS AF 2.7.3.9723 software and analyzed using ImageJ.

\section{Results}

Molecular investigations performed on the brain yielded positive results for TBEV. The metagenomic approach allowed to reveal and identify TBEV as the unique viral pathogen in the brain. An almost complete genome sequence with a length of 10,897 nucleotides was obtained (sequences available in GenBank under the accession numbers OM084948). The coding region extended from the nucleotide position 114 to 10,358 and corresponded to a polyprotein of 3414 amino acids. From the BLAST carried out with the consensus sequence of the genome towards the sequences available in the database (https://blast.ncbi.nlm.nih.gov/Blast.cgi), the highest similarity (99.17\%) was identified with the TBEV AS33 (GQ266392) strain isolated from ticks in Germany in 2005. As previously seen for the German strains named Salem (FJ572210) and AS33 (GQ266392), the virus genome presents a deletion of 43 nucleotides in the untranslated region (NTR) of polyadenosine in relation to reference strain Neudoerfl (U27495) [45]. At the amino acid level, there were 18 differences scattered throughout the genome compared to the AS33 strain, while 37 compared to the Neudoerfl strain. Amino acids differences observed from the comparison of E and NS5 proteins of the 21RS1767, AS33 and Neudoerfl viruses are summarized in table S1 (Neudoerfl numbering for nucleotide positions was considered). Interestingly, E protein shows 3 differences in antigenic domains I and II [46]; in particular 21RS1767 shows Glu (E) at position 331 of the central domain (I) and Thr (T) at position 361 of the dimerization domain (II) like Neudoerfl virus, whereas at position 408 (central domain (I)) shows Ile (I) like AS33 virus. At position 761 in the E stem anchor, 21RS1767 virus shows an Ile (I) whereas AS33 and Neudoerfl show a Leu (L). NS5 protein shows a total of ten differences; in particular five are within the N-terminal RNA methyltransferase (MTase) domain and one in the RdRp catalytic domain. Position 2532 and 2559 show $\operatorname{Arg}(\mathrm{R})$ and Glu (E) respectively like Neudoerfl, whereas 2562, 2619 and 2764 shows the same residues present in AS33 (see table 2 for details). Position 3297 in RdRp catalytic domain shows a Val (V) for both 21RS1767 and AS33, whereas Neudoerfl shows Ala (A). Mutations in the $\mathrm{E}$ and NS protein involved in TBE neuroinvasiveness and neurovirulence previously listed in the review of the Kellman et al (2018) have not been detected [3].

Based on phylogenetic analysis of gene E, sample 21RS1767 grouped within the European subtype (Figure 1); the highest percentage of nucleotide similarity (100\%) was found to be with the partial sequence (520 nt) of a TBEV obtained from a pool of ticks collected in 2018 on Monte Calisio in Val d'Adige (TN) (Genbank accession number MN746771), about $100 \mathrm{~km}$ far from Modolo, which was also presenting the Ile (I) at position 408 [46]. 
5 of 12

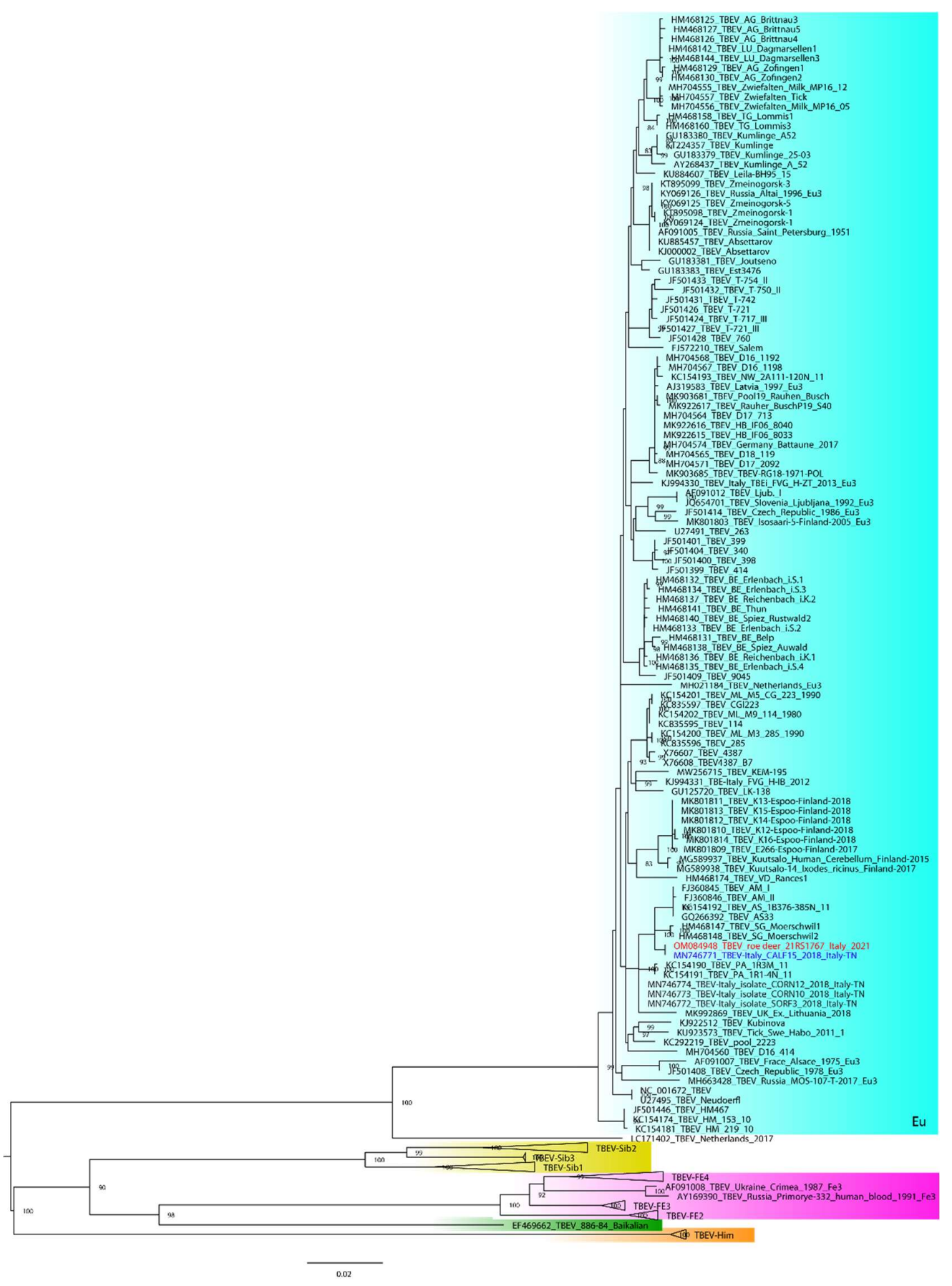


Figure 1. Maximum likelihood phylogenetic tree of TBEV E gene. In red the virus 21RS1767_TBEV_Italy_2021 amplified from the roe deer's brain. European (TBEV-Eu), Siberian (TBEV-Sib), Far Eastern (TBEV-FE), Baikalian (TBEV-Bkl) e Himalayan (TBEV-Him) are grouped and highligthed respectively in ligth blue, yellow, purple, green and orange. The highest percentage of nucleotide similarity $(100 \%)$ was found with the partial sequence (520 nt) of a TBEV obtained from a pool of ticks collected in 2018 on Monte Calisio in Val d'Adige (TN) (virus marked in blue).

TBEV and Babesia spp. were not found in ticks. Four ticks out of seventeen harbored $A$. phagocytophylum, two tested positive for $R$. helvetica and two were co-infected by two pathogens: one by A. phagocytophilum and R. helvetica and another by A. phagocytophilum and B. afzelii.

At histological examination, the midbrain showed a moderate, multifocal encephalitis characterized by perivascular cuffs and neuropil infiltrates of lymphocytes and histiocytes mixed with fewer eosinophils and rare neutrophils, in both grey (Figure 2) and white matter. Moreover, gliosis, neuronal chromatolysis and rare microglial nodules were observed in the grey matter. The presence of active microglia was further confirmed through immunofluorescence staining for the marker Iba1 (Figure 3). The increased number of active immune cells in the midbrain of the infected roe deer has been observed by comparing the number of Iba1 cells in a non-infected, control roe deer brain.

Diagnostic tests for L. monocytogenes and T. gondii yielded negative results.

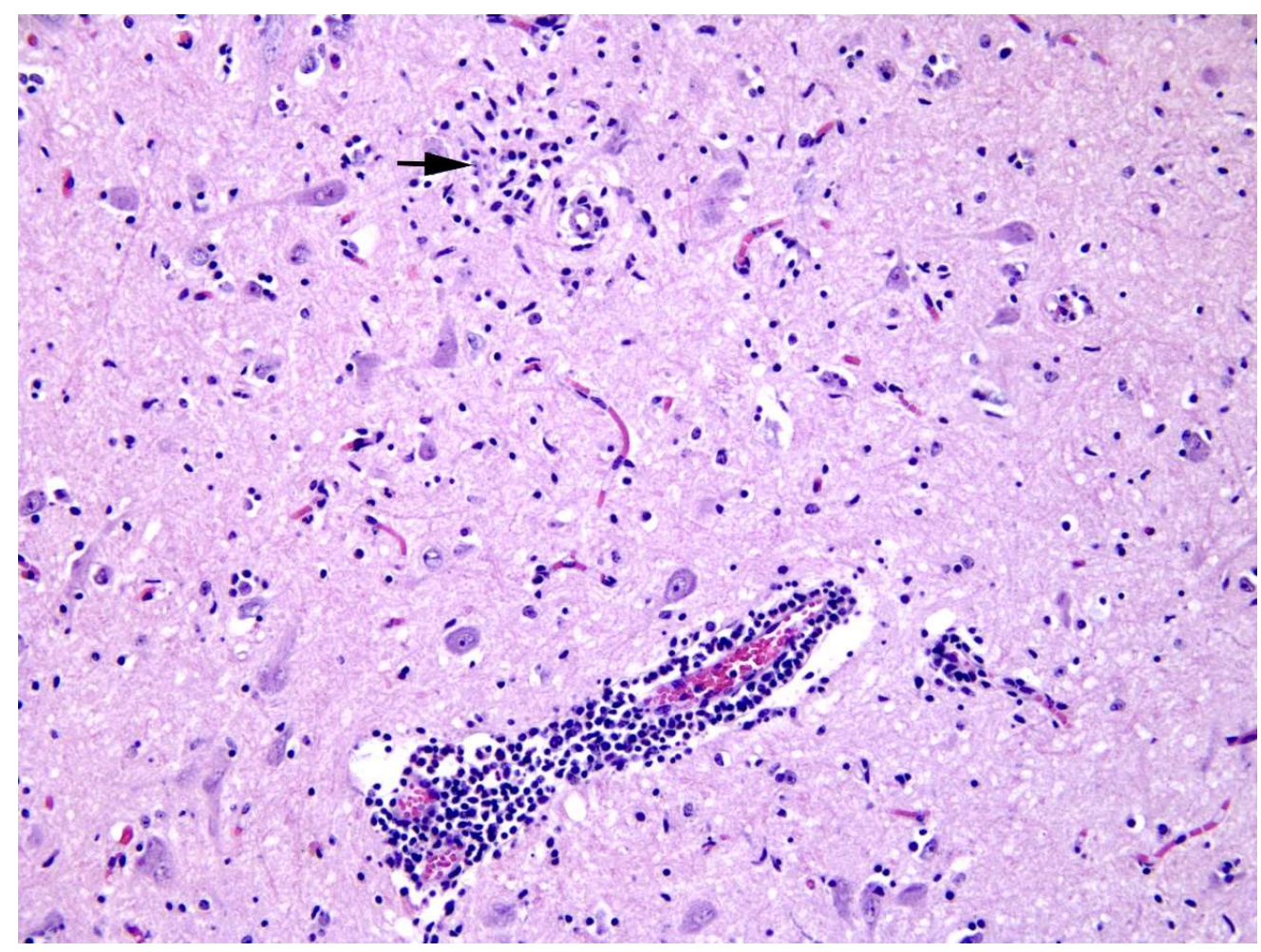

Figure 2. Histologic section of the midbrain (grey matter) stained with H\&E. A perivascular space (lower center) is infiltrated by lymphocytes and histiocytes; the surrounding neuroparenchyma is hypercellular due to increased numbers of glial cells, including microglia, that occasionally form nodules (arrow) or arrange near the body of neurons (satellitosis). 


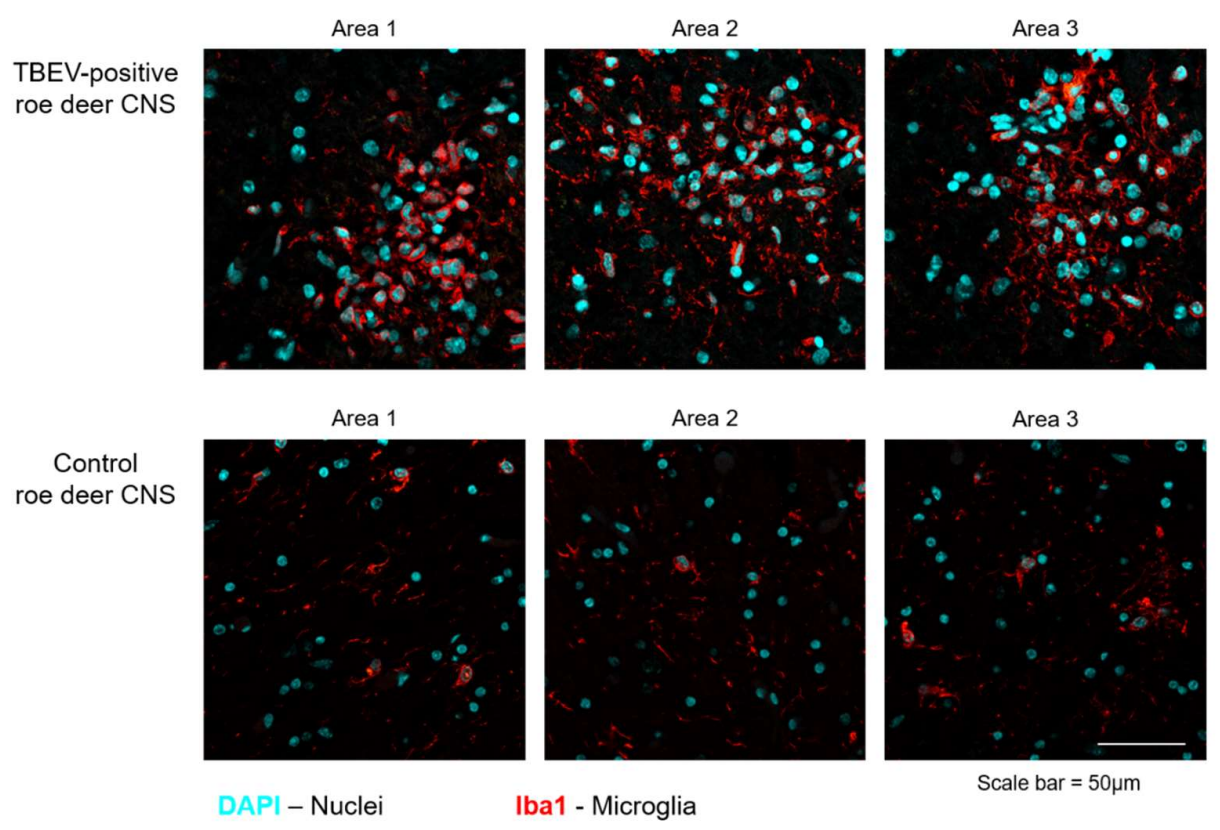

Figure 3. Representative staining of Iba1+ cells (microglia, red) in TBEV-positive and control roe deer midbrain. Nuclei are stained with DAPI, light blue. Scale bar: $50 \mu \mathrm{m}$.

\section{Discussion}

We herewith described the clinical, pathological and virological findings of a symptomatic roe deer infected by a TBEV from a known endemic area of North-Eastern Italy. The TBEV responsible for this case was characterized as an European subtype with the highest percentage of nucleotide similarity $(100 \%)$ at the E gene with the partial sequence (520 nt) of a TBEV (MN746771) obtained from a pool of ticks collected in Trento province in 2018, a location about $100 \mathrm{~km}$ far from the case described herein [46].

Histological findings in the roe deer's midbrain were supportive of a neurotropic virus infection, although we did not observe any extensive neuronal necrosis and neuronophagy as described in human or canine TBE [47]. Notably, we observed the activation of microglia in the midbrain of the infected animal, as depicted by histological analysis and immunofluorescence for the specific marker Iba1. Unfortunately, we did not find any viral protein by immunofluorescence, as the specimen material was too small and probably not representative of the ongoing TBEV infection that was actually identified by realtime RT-PCR. Given the lower presence of eosinophils and neutrophils in the infiltrate, we further ruled out the presence of co-infections through immunohistochemistry tests for T. gondii and L. monocytogenes.

Molecular investigations did not reveal the presence of mutations in the $\mathrm{E}$ and NS protein notably involved in TBE neuroinvasiveness and neurovirulence previously identified $[3,48]$. It is however worth to mention the presence of amino acid differences compared to AS33 and Neudoerfl strains in the antigenic domains (I and II), a region involved in the viral membrane fusion with the host cell: a finding deserving further investigation.

Our report leads to the inclusion of TBEV in the frame of a differential diagnosis of clinical encephalitis in roe deer, especially within and around known foci. In a public 
health perspective, the case herein described, as well as those quoted in the literature, cannot be used as an alert. Actually, even in case of emergence of TBEV, clinical episodes in animals, if present, would be unfortunately preceded by far by cases in humans [49]. Nevertheless, cases in animals should be monitored and framed in a consistently mutating ecopathological scenario. Actually, like other large wild and domestic mammals, roe deer seems not to play a direct role in the maintenance of TBEV, because generally the level of viraemia after natural infection is too low to infect ticks and, therefore the absence of TBEV in the ticks collected from the roe deer's carcass was not surprising [50-51]. Nevertheless, roe deer play a role in the TBEV ecology, being a key host in granting survival and abundance of $I$. ricinus populations [52]. It is worth to note that in North-Eastern Italy, and more broadly in the Alps, ungulates have been increasing for decades, in parallel to the progressive depopulation and loss of human activities. Since fawns and yearlings (as the case presented herein) are the age categories more than others accounting for an incomplete immunity to microparasites as TBEV and heavy infestation by all active stages of $I$. ricinus [13], and studies in other species (e.g. horses) suggest declining levels of TBEV antibodies following passive transfer from foals to yearlings, a high roe deer population turnover in conjunction with environmental conditions favorable to ticks may lead to unpredictable variations in the ecology of the disease. [53]. Considering all of the above, although referring to a single case, our report stresses the importance to flank information on host population dynamics to pathogen knowledge.

Supplementary Materials: TableS1, Video S1 and Video S2 are available online at www.mdpi.com/xxx/s1 .

Author Contributions: Conceptualization, C.V.C. and G.D.R.; methodology, C.V.C..; software, G.Z., A.M.; formal analysis, C.V.C., F.O., A.M., G.Z., G.F., M.C.; investigation, M.V., G.F., M.C., P.D., S.R., F.T, S.G; data curation, G.D.R; writing-original draft preparation, G.D.R. and C.V.C.; writingreview and editing: G.D.R, P.D.B., I.M., A.M., G.F.; supervision, C.V.C., P.D.B, I.M.; project administration, C.V.C..; funding acquisition, G.D.R. All authors have read and agreed to the published version of the manuscript.

Funding: This work has been partially supported by the project IZSVE RC 08/2020, funded by the Italian Ministry of Health.

Data Availability Statement: Consensus sequence of TBEv was deposided in GenBank under accession number OM084948. MiSeq raw sequencing data are available at the NCBI Sequence Read Archive (SRA) under accession number PRJNA793165.

Acknowledgments: Authors are grateful to the Belluno Provincial Police (concerning the case described in this paper, a special mention is due to Daniele Comiotto), for their consistent and fruitful effort in passive surveillance. We wish to thank Antonella Lettini for support in differential diagnosis, Erica Melchiotti for immunohistochemical analysis and Luca Lunardi for support in video editing.

Conflicts of Interest: The authors declare no conflict of interest. The funders had no role in the design of the study; in the collection, analyses, or interpretation of data; in the writing of the manuscript; or in the decision to publish the results.

\section{Appendix A}

Raw data were filtered by removing: a) reads with more than $10 \%$ of undetermined ("N") bases; b) reads with more than 100 bases with Q score below 7; c) duplicated pairedend reads. Remaining reads were clipped from Illumina adaptors Nextera XT with scythe v0.991 (https:/github.com/vsbuffalo/scythe) and trimmed with sickle v1.33 (https://github.com/najoshi/sickle). Reads shorter than 80 bases or unpaired after previous filters were discarded. Taxonomic assignment of high-quality reads was carried out using Basic local alignment search tool (BLAST 2.10.0+) alignment against the integrated NT 
database (version 23 February 2020) and diamond v0.9.17 alignment against the integrated NR database (version 23 February 2020) [54]. Alignment hits with e-values greater than $1 \times 10^{-3}$ were discarded. Taxonomical level of each read was determined by the lowest common ancestor (LCA)-based algorithm that was implemented in MEGAN v6.18.50 [55]. For the reconstruction of the complete genome, reads taxonomically classified as belonging to TBE were selected and de novo assembled using IDBA-UD v1.1.1 with the multikmer approach using a minimum value of 24, a maximum value of 124 and an inner increment of 10 [56]. The longest contig produced was blasted online, resulting in $>99 \%$ similarity with the Genbank record "Tick-borne encephalitis virus isolate AS33, complete genome" (accession number GQ266392.1), which was subsequently used for a referencebased assembly. High quality reads were aligned against the chosen reference genome using BWA v0.7.12 and standard parameters [57]. Alignments were processed with SAMtools v1.6 to convert them in BAM format and sort them by position [58]. SNPs were called using LoFreq v2.1.2 [59]. According to LoFreq usage recommendations, the alignment was first processed with Picard-tools v2.1.0 (http://broadinstitute.github.io/picard/) and GATK v3.5 in order to correct potential errors, realign reads around indels and recalibrate base quality [60]. LoFreq was then run on fixed alignment with option "--call-indels" to produce a vcf file containing both SNPs and indels. From the final set of variants, indels with a frequency lower than $50 \%$ and SNPs with a frequency lower than $25 \%$ were discarded. To produce the consensus sequence we changed the reference genome in agreement with the following rules: a) for a position $j$, if coverage was not enough to reliably call variants we added an "N" base; b) for a position $j$, if coverage was enough to reliably call variants but no SNP were called, we added reference genome base at position $\mathrm{j}$; c) for a position $\mathrm{j}$, if coverage was enough to reliably call variants and at least one SNP were called, we added the nucleotide using the IUPAC nucleotide code (http://www.bioinformatics.org/sms/iupac.html) according to the bases present. Finally, high quality reads were re-aligned with BWA against the consensus sequence produced; we performed a visual inspection of the alignment with tablet v1.14.10.21 and, if required, manually revised the consensus sequence based on this alignment [61].

\section{References}

1. Lindquist, L.; Vapalahti, O. Tick-borne encephalitis. Lancet 2008; 371, 1861-71, doi.org/10.1016/S0140-6736(08)60800-4.

2. Kovalev, S. Y.; Mukhacheva, T. A. Reconsidering the classification of tick-borne encephalitis virus within the Siberian subtype gives new insights into its evolutionary history. Infect. Genet. Evol. 2017, 55, 159-165, doi: 10.1016/j.meegid.2017.09.014.

3. Kellman, E. M.; Offerdahl, D.K.; Melik, W.; Bloom, M.E. Viral Determinants of Virulence in Tick-Borne Flaviviruses. Viruses, 2018, 10, 329, doi: 10.3390/v10060329.

4. Gritsun TS, Lashkevich VA, Gould EA. Tick-borne encephalitis. Antiviral Res. 2003, 57, 129-146, doi: 10.1016/s01663542(02)00206-1.

5. Ruzek, D.; Avšič Županc, T. Borde, J.; Chrdle, A.,; Eyer, L.; Karganova, G.; Kholodilov, I.; Knap, N.; Kozlovskaya, L.; Matveev, A.; Miller, A. D.; Osolodkin, D. I.; Överby, A. K.; Tikunova, N.; Tkachev, S.; Zajkowska, J. Tick-borne encephalitis in Europe and Russia: Review of pathogenesis, clinical features, therapy, and vaccines. Antiviral Res. 2019, 164, 23-51, doi.org/10.1016/j.antiviral.2019.01.014.

6. Süss, J. Epidemiology and ecology of TBE relevant to the production of effective vaccines. Vaccine 2003, 21, 19-35, doi:10.1016/S0264-410X(02)00812-5.

7. Cisak, E.; Wójcik-Fatla, A.; Zając, V.; Sroka, J.; Buczek, A.; Dutkiewicz, J. Prevalence of tick-borne encephalitis virus (TBEV) in samples of raw milk taken randomly from cows, goats and sheep in eastern Poland. Ann Agric Environ Med. 2010, 17, 283-286.

8. Nuttall, P. A.; Labuda, M. Dynamics of infection in tick vectors and at the tick-host interface. Adv. virus Res. 2003, 60, 232272, doi.org/10.1016/s0065-3527(03)60007-2.

9. Labuda, M.; Kozuch, O.; Zuffová, E.; Elecková, E.; Hails, R. S.; Nuttall, P. A. Tick-Borne Encephalitis Virus Transmission between Ticks Cofeeding on Specific Immune Natural Rodent Hosts. Virology 1997, 235, 138-143, doi.org/10.1006/viro.1997.8622.

10. Labuda, M.; Randolph, S.E. Survival strategy of tick-borne encephalitis virus: cellular basis and environmental determinants. Zentralbl Bakteriol. 1999, 289, 513-24. doi: 10.1016/s0934-8840(99)80005-x. 
11. Randolph, S.E.; Gern, L.; Nuttall, P. A. Co-feeding ticks: Epidemiological significance for tick-borne pathogen transmission, Parasitol Today 1996, 12, 472-479, doi.org/10.1016/S0169-4758(96)10072-7.

12. Slovák, M.; Kazimírováa, M.; Siebenstichová, M.; Ustaníková, K.; Klempa, B.; Gritsun, T.; Gould, E. A.; Nuttall, P. A. Survival dynamics of tick-borne encephalitis virus in Ixodes ricinus ticks. Ticks tick borne Dis. 2014, 5, 962-969, doi: 10.1016/j.ttbdis.2014.07.019.

13. Jaenson, T. G. T; Petersson, E. H.; Jaenson, D.; Kindberg, J.; Pettersson, J. H.; Hjertqvist, M.; Medlock, J. M.; Bengtsson, H. The importance of wildlife in the ecology and epidemiology of the TBE virus in Sweden: Incidence of human TBE correlates with abundance of deer and hares. Parasit Vectors 2018,11, 477, doi: 10.1186/s13071-018-3057-4.

14. Randolph, S. E.; Green, R. M.; Peacey, M. F.; Rogers, D. J. Seasonal synchrony: the key to tick-borne encephalitis foci identified by satellite data. Parasitology 2000, 121, 15-23, doi: 10.1017/S0031182099006083.

15. Rosà, R.; Tagliapietra, V.; Manica, M.; Arnoldi, D.; Hauffe, H. C.; Rossi, C.; Rosso, F.; Henttonen, H.; Rizzoli, A. Changes in host densities and co-feeding pattern efficiently predict tick-borne encephalitis hazard in an endemic focus in northern Italy. Int. J. Parasitol. 2019, 49, 779-787, doi.org/10.1016/j.ijpara.2019.05.006.

16. Hekrlová, A.; Kubíček, O.; Lány, P.; Rosenbergová, K.; Schánilec, P. Tick-borne encephalitis in dogs: application of "nested real-time RT-PCR" for intravital virus detection. Berl Munch Tierarztl Wochenschr. 2015, 128, 397-401.

17. Beckmann, K.; Oevermann, A.; Golini, L.; Steffen, F.; Kircher, P.R.; Carrera, I. MRI findings in a case of canine tick born meningoencephalomyelitis. Schweiz. Arch. Tierheilkd. 2014, 156, 395-399, doi: 10.1024/0036-7281/a000612.

18. Pfeffer, M.; Dobler, G. Tick-borne encephalitis virus in dogs - Is this an issue?. Parasit Vectors 2011, 4, 59, doi: 10.1186/17563305-4-59.

19. Leschnik, M.W.; Kirtz, G.C.; Thalhammer, J. G. Tick-borne encephalitis (TBE) in dogs. Int J Med Microbiol. 2002, 291, 66-69, doi: 10.1016/s1438-4221(02)80014-5.

20. Andersson, E.; Kendall, A.; Url, A.; Auer, A.; Leschnik, M. The first RT-qPCR confirmed case of tick-borne encephalitis in a dog in Scandinavia. Acta Vet Scand 2020, 62, 51, doi: 10.1186/s13028-020-00550-2.

21. Klaus, C.; Hörügel, U.; Hoffmann, B.; Beer, M. Tick-borne encephalitis virus (TBEV) infection in horses: Clinical and laboratory findings and epidemiological investigations. Vet. Microbiol. 2013, 163, 368-372, doi: 10.1016/j.vetmic.2012.12.041.

22. Fouché, N.; Oesch, S.; Ziegler, U.; Gerber, V. Clinical presentation and laboratory diagnostic work-up of a horse with tickborne encephalitis in Switzerland. Viruses 2021, 13, 1474, doi: 10.3390/v13081474.

23. Süss, J.; Gelpi, E.; Klaus, C.; Bagon, A.; Liebler-Tenorio, E. M.; Budka, H.; Stark, B.; Müller, W.; Hotzel, H. Tickborne encephalitis in naturally exposed monkey (Macaca sylvanus). Emerg Infect Dis. 2007, 13, 905-907, doi: 10.3201/eid1306.061173.

24. Klaus, C.; Hoffmann, B.; Beer, M.; Müller, W.; Stark, B.; Bader, W.; Stiasny, K.; Heinz, F. X.; Süss, J. Seroprevalence of tickborne encephalitis (TBE) in naturally exposed monkeys (Macaca sylvanus) and sheep and prevalence of TBE virus in ticks in a TBE endemic area in Germany. Ticks Tick Borne Dis. 2010, 1, 141-144, doi: 10.1016/j.ttbdis.2010.06.001.

25. Böhm, B.; Schade, B.; Bauer, B.; Hoffmann, B.; Hoffmann, D.; Ziegler, U.; Beer, M.; Klaus, C.; Weissenböck, H.; Böttcher, J. Tick-borne encephalitis in a naturally infected sheep. BMC Vet Res. 2017, 13, 267, doi: 10.1186/s12917-017-1192-3.

26. Zindel, W.; Wyler, R. Tick-borne encephalitis in a goat in lower Prätigau. Schweiz Arch Tierheilkd. 1983, 125, 383-386.

27. Bagó, Z.; Bauder, B.; Kolodziejek, J.; Nowotny, N.; Weissenböck, H. Tickborne encephalitis in a mouflon (Ovis ammon musimon). Vet Rec. 2002, 150, 218-220, doi: 10.1136/vr.150.7.218.

28. Salat. J.; Ruzek, D. Tick-borne encephalitis in domestic animals. Acta Virol. 2020, 64, 226-232, doi:10.4149/av 2020 212

29. Kiffner, C.; Lödige, C.; Alings, M.; Vor, T.; Rühe, F. Abundance estimation of Ixodes ticks (Acari: Ixodidae) on roe deer (Capreolus capreolus). Exp Appl Acarol. 2010, 52(1), 73-84, doi.org/10.1007/s10493-010-9341-4.

30. Kriz, B.; Daniel, M.; Benes, C.; Maly, M. The role of game (wild boar and roe deer) in the spread of tick-borne encephalitis in the Czech Republic. Vector Borne Zoonotic Dis. 2014, 14, 801-807, doi:10.1089/vbz.2013.1569.

31. Gerth, H. J.; Grimshandl, D.; Stage, B.; Döller, G.; Kunz, C. Roe deer as sentinels for endemicity of tick-borne encephalitis virus. Epidemiol. Infect. 1995, 115, 355-365, doi.org/10.1017/s0950268800058477.

32. Carpi, G.; Cagnacci, F.; Neteler, M.; Rizzoli, A. Tick infestation on roe deer in relation to geographic and remotely sensed climatic variables in a tick-borne encephalitis endemic area. Epidemiol. Infect. 2008, 136, 1416-1424, doi.org/10.1017/S0950268807000039.

33. Knap, N.; Avšič-Županc, T. Correlation of TBE incidence with red deer and roe deer abundance in Slovenia. PloS One 2013, 8,66380, doi.org/10.1371/journal.pone.0066380.

34. Pugliese, A.; Rosà, R. Effect of host populations on the intensity of ticks and the prevalence of tick-borne pathogens: how to interpret the results of deer exclosure experiments. Parasitology 2008, 135, 1531-1544. doi.org/10.1017/S003118200800036X.

35. Rizzoli, A.; Hauffe, H. C.; Tagliapietra, V.; Neteler, M.; Rosà, R. Forest structure and roe deer abundance predict tick-borne encephalitis risk in Italy. PloS One 2009, 4, e4336, doi.org/10.1371/journal.pone.0004336.

36. Król, N.; Chitimia-Dobler, L.; Dobler, G.; Karliuk, Y.; Birka, S.; Obiegala, A.; Pfeffer, M. Tick burden on European roe deer (Capreolus capreolus) from Saxony, Germany, and detection of tick-borne encephalitis virus in attached ticks. Parasitol. Res. 2020, 119, 1387-1392. doi.org/10.1007/s00436-020-06637-z.

37. De Benedictis, P.; De Battisti C.; Dacheux, L.; Marciano, S.; Ormelli, S.; Salomoni, A.; Caenazzo, S. T.; Lepelletier, A.; Bourhy, H.; Capua, I.; Cattoli, G. Lyssavirus detection and typing using pyrosequencing. J Clin Microbiol. 2011, 49, 1932-1938, doi:10.1128/JCM.02015-10. 
38. OIE manual rabies chapter 3. 1. 17, 2018 https://www.oie.int/fileadmin/Home/eng/Health_standards/tahm/3.01.17_RABIES.pdf

39. Schwaiger, M.; Cassinotti, P. Development of a quantitative real-time RT-PCR assay with internal control for the laboratory detection of tick borne encephalitis virus (TBEV) RNA. J. Clin. Virol. 2003, 27, 136-145. doi.org/10.1016/s1386-6532(02)00168$\underline{3}$.

40. Da Rold, G.; Ravagnan, S.; Soppelsa, F.; Porcellato, E.; Soppelsa, M.; Obber, F.; Citterio, C. V.; Carlin, S.; Danesi, P.; Montarsi, F.; Capelli, G. Ticks are more suitable than red foxes for monitoring zoonotic tick-borne pathogens in northeastern Italy. Parasit Vectors. 2018, 11 , 137, doi.org/10.1186.

41. Katoh, K.; Standley, D.M. MAFFT Multiple Sequence Alignment Software Version 7: Improvements in Performance and Usability, Mol Biol Evol. 2013, 30, 772-780, doi.org/10.1093/molbev/mst010.

42. Dai, X.; Shang G.; Lu, S.; Yang, J.; Xu, J. A new subtype of eastern tick-borne encephalitis virus discovered in Qinghai-Tibet Plateau, China. Emerg Microbes Infect. 2018, 25, 74, doi: 10.1038/s41426-018-0081-6.

43. Ecker, M.; Allison, S.L.; Meixner, T.; Heinz, F. X. Sequence analysis and genetic classification of tick-borne encephalitis viruses from Europe and Asia. J Gen Virol. 1999, 80, 179-185, doi: 10.1099/0022-1317-80-1-179.

44. Kupča, A.M.; Essbauer, S.; Zoeller, G.; de Mendonça, P. G.; Brey, R.; Rinder, M.; Pfister, K.; Spiegel, M.; Doerrbecker, B.; Pfeffer, M.; Dobler, G. Isolation and molecular characterization of a tick-borne encephalitis virus strain from a new tickborne encephalitis focus with severe cases in Bavaria, Germany. Ticks Tick Borne Dis. 2010, 1, 44-51, doi: 10.1016/j.ttbdis.2009.11.002.

45. Rey, F.A.; Heinz, F.X.; Mandl, C.; Kunz, C.; Harrison, S. C. The envelope glycoprotein from tick-borne encephalitis virus at 2 A resolution. Nature, 1995, 375, 291-298, doi:10.1038/375291a0.

46. Alfano, N.; Tagliapietra, V.; Rosso, F.; Ziegler, U.; Arnoldi, D.; Rizzoli, A. Tick-borne encephalitis foci in northeast Italy revealed by combined virus detection in ticks, serosurvey on goats and human cases. Emerg. microbes Infect. 2020, 9, 474484, doi: 10.1080/22221751.2020.1730246.

47. Weissenböck, H.; Suchy, A.; Holzmann, H. Tick-borne encephalitis in dogs: neuropathological findings and distribution of antigen. Acta Neuropathol. 1998, 95, 361-366, doi.org/10.1007/s004010050811.

48. Pulkkinen, L.; Butcher, S. J.; Anastasina, M. Tick-Borne Encephalitis Virus: A Structural View. Viruses 2018, 10, 350, doi.org/10.3390/v10070350.

49. Rezza, G.; Farchi, F.; Pezzotti, P.; Pezzotti, P.; Ruscio, M.; Lo Presti, A.; Ciccozzi, M.; Mondardini, V.; Paternoster, C.; Bassetti, M.; Merelli, M.; Scotton, P. G.; Luzzati, R.; Simeoni, J.; Mian, P.; Mel, R.; Carraro, V.; Zanin, A.; Ferretto, R.; Francavilla, E.; TBE Virology Group Tick-borne encephalitis in north-east Italy: a 14-year retrospective study, January 2000 to December Euro Surveill. 2015, 20, 10, doi:10.2807/1560-7917.ES.2015.20.40.30034.

50. Jaenson, T.G.; Hjertqvist, M.; Bergström, T.; Lundkvist, A. Why is tick-borne encephalitis increasing? A review of the key factors causing the increasing incidence of human TBE in Sweden. Parasit Vectors 2012, 5, 184, doi.org/10.1186/1756-3305$\underline{5-184}$.

51. Paulsen, K.M.; Das Neves, C.G., Granquist, E.G.; Madslien, K.; Stuen, S.; Pedersen, B. N.; Vikse, R.; Rocchi, M.; Laming, E.; Stiasny, K.; Andreassen, Å. K. Cervids as sentinel-species for tick-borne encephalitis virus in Norway - A serological study. Zoonoses Public Health. 2020, 67, 342-351, doi:10.1111/zph.12675

52. Opalińska, P.; Wierzbicka, A.; Asman, M.; Rączka, G.; Dyderski, M. K.; Nowak-Chmura, M. Fivefold higher abundance of ticks (Acari: Ixodida) on the European roe deer (Capreolus capreolus L.) forest than field ecotypes. Sci Rep 2021, 11, 10649, doi.org/10.1038/s41598-021-90234-2.

53. Pautienius, A.; Armonaite, A.; Simkute, E.; Zagrabskaite, R.; Buitkuviene, J.; Alpizar-Jara, R.; Grigas, J.; Zakiene, I.; Zienius, D.; Salomskas, A.; Stankevicius, A. Cross-Sectional Study on the Prevalence and Factors Influencing Occurrence of TickBorne Encephalitis in Horses in Lithuania. Pathogens 2021, 10, 140, doi.org/10.3390/pathogens10020140

54. Buchfink, B.; Xie, C.; Huson, D. H. Fast and sensitive protein alignment using DIAMOND. Nat Methods 2015, 12, 59-60, doi.org/10.1038/nmeth.3176.

55. Huson, D.H.; Beier, S.; Flade, I.; Górska, A.; El-Hadidi, M.; Mitra, S.; Ruscheweyh, H. J.; Tappu, R. MEGAN Community Edition - Interactive Exploration and Analysis of Large-Scale Microbiome Sequencing Data. PLoS Comput Biol 2016, 12, e1004957, doi.org/10.1371/journal.pcbi.1004957.

56. Peng, Y.; Leung, H. C.; Yiu, S. M.; Chin, F. Y. IDBA-UD: a de novo assembler for single-cell and metagenomic sequencing data with highly uneven depth, Bioinformatics 2012, 28, 1420-1428, doi.org/10.1093/bioinformatics/bts174.

57. Li, H.; Durbin, R. Fast and accurate long-read alignment with Burrows-Wheeler transform. Bioinformatics 2010, 26, 589595, doi.org/10.1093/bioinformatics/btp698.

58. Li, H.; Handsaker, B.; Wysoker, A.; Fennell, T.; Ruan, J.; Homer, N.; Marth, G.; Abecasis, G.; Durbin, R.; 1000 Genome Project Data Processing Subgroup The Sequence Alignment/Map format and SAMtools, Bioinformatics 2009, 25, 2078-2079, doi.org/10.1093/bioinformatics/btp352.

59. Wilm, A.; Aw, P. P.; Bertrand, D.; Yeo, G. H.; Ong, S. H.; Wong, C. H.; Khor, C. C.; Petric, R.; Hibberd, M. L.; Nagarajan, N. LoFreq: a sequence-quality aware, ultra-sensitive variant caller for uncovering cell-population heterogeneity from highthroughput sequencing datasets, Nucleic Acids Res. 2012, 40, 11189-11201, doi.org/10.1093/nar/gks918. 
60. McKenna, A.; Hanna, M.; Banks, E.; Sivachenko, A.; Cibulskis, K.; Kernytsky, A.; Garimella, K.; Altshuler, D.; Gabriel, S.; Daly, M.; DePristo, M. A. The Genome Analysis Toolkit: a MapReduce framework for analyzing next-generation DNA sequencing data. Genome Res. 2010, 20, 1297-1303, doi: 10.1101/gr.107524.110.

61. Milne, I.; Bayer, M.; Cardle, L.; Shaw, P.; Stephen, G.; Wright, F.; Marshall, D. Tablet-next generation sequence assembly visualization, Bioinformatics 2010, 26, 401-402, doi.org/10.1093/bioinformatics/btp666. 